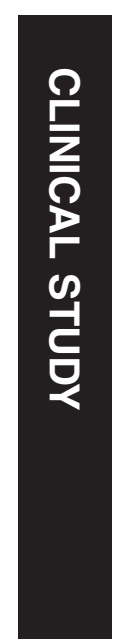

\section{Depression and anxiety in dry eye disease: a systematic review and meta-analysis}

\begin{abstract}
Aim To evaluate the association of dry eye disease (DED) with depression and anxiety. Patients and methods We conducted a systematic review and meta-analysis of studies that reported the prevalence, incidence and/or severity grading of depression and/or anxiety in DED patients and healthy controls. We searched MEDLINE, EMBASE, PsycINFO, ClinicalTrials.gov, and World Health Organization International Clinical Trials Registry Platform for relevant studies. Results Twenty-two eligible studies consisted of 2980026 patients were analyzed. DED was associated with an increased prevalence of depression (summary odds ratio $(O R)=2.92,95 \%$ CI: 2.13-4.01, $P<0.00001$ ) and anxiety $(\mathrm{OR}=2.80,95 \% \mathrm{CI}: 2.61-3.02$, $P<0.00001)$. The depression score (standardized mean difference $(\mathrm{SMD})=\mathbf{0 . 8 1}$, 95\% CI: $0.48-1.15, P<0.00001)$ and anxiety score $(S M D=0.37,95 \%$ CI: $0.10-0.64$, $P=0.007)$ were higher in DED patients than in controls. Subgroup analyses revealed that the prevalence and severity of depression are greatest in primary Sjogren's syndrome patients. No study reported the incidence. Conclusion Depression and anxiety are more prevalent in DED patients than in controls. Among patients with DED, those suffering from primary Sjogren's syndrome have higher prevalence and severity of depression.

Eye (2016) 30, 1558-1567; doi:10.1038/eye.2016.186; published online 12 August 2016
\end{abstract}

${ }^{1}$ Department of Ophthalmology and Visual Sciences, The Chinese University of Hong Kong, Hong Kong, China

\section{${ }^{2}$ Department of Ophthalmology, Tuen Mun Eye Center and Tuen Mun Hospital, Hong Kong, China}

${ }^{3}$ Department of Ophthalmology and Visual Sciences, Prince of Wales Hospital \& Alice Ho Liu Ling Nethersole Hospital, Hong Kong, China

Correspondence: AL Young, Department of Ophthalmology and Visual Sciences, Prince of Wales Hospital \& Alice Ho Liu Ling Nethersole Hospital, Hong Kong 00852, China Tel: +852 26322878; Fax: +85226482943 . E-mail: youngla@ha.org.hk

Received: 4 March 2016 Accepted in revised form: 16 June 2016 Published online: 12 August 2016

\section{Introduction}

Dry eye disease (DED) is one of the most common conditions encountered in an ophthalmology clinic. ${ }^{1}$ DED is a multifactorial disease of the tears and ocular surface that results in tear film instability, ocular discomfort, and visual disturbance. ${ }^{1}$ Symptoms of DED include constant ocular irritation, foreign body sensation, and blurred vision, which lead to negative impact on the patients' daily life and social functioning, making DED an important public health problem. ${ }^{2,3}$ However, there is discrepancy between the ocular signs and symptoms of DED,, 45 and the symptoms of DED are more aligned to non-ocular conditions than to the tear film parameters. ${ }^{6}$ DED has recently been associated with other chronic pain conditions and may share genetic susceptibility with depression. ${ }^{7-9}$

A number of epidemiological studies have investigated the association of DED with depression and anxiety. These studies have postulated potential explanations for their association, by looking into the prevalence of mental disorders in DED patients or vice versa. However, the prevalence of DED varies with disease definition, sex, age, lifestyle, and geography. ${ }^{10}$ In order to summarize the results and explore the possible explanations, we conducted a systematic review and metaanalysis to evaluate the association between different forms of DED with depression and anxiety.

\section{Materials and methods}

\section{Eligibility criteria for study inclusion}

In this systematic review, we followed the guidelines for reporting Meta-Analysis of Observational Studies in Epidemiology (MOOSE). ${ }^{11}$ The MOOSE checklist is provided in Supplementary Appendix 1. We included all studies that fulfilled the following criteria: studies with documented criteria in their study design in defining patients as having DED, depression, or anxiety; studies examining the outcomes on the prevalence, incidence, or severity of depression and/or anxiety in DED and non-DED patients; and studies published in English. We included all types of studies. We excluded studies lacking a healthy control 
group, studies lacking sufficient details to define whether the patient is suffering or not suffering from DED, depression or anxiety, and studies with outcomes on cognitive or neuropsychiatric impairment that is not specific to depression or anxiety.

\section{Search for eligible studies}

We performed the literature search in MEDLINE, EMBASE, PsycINFO via the OVID platform, ClinicalTrials.gov (www.clinicaltrials.gov) and World Health Organization International Clinical Trials Registry Platform (WHO ICTRP). We described our detailed search strategy in Supplementary Appendix 2 together with the number of results yielded. We then manually searched the references of the original studies and reviews to identify any potential studies omitted by our search strategy. Where published information was unclear or inadequate, we contacted the corresponding authors for more information. We conducted the final search on 6 May 2015 in all databases.

\section{Study inclusion}

We followed the Cochrane Handbook for Systematic Review of Interventions to select studies to be reviewed based on our inclusion and exclusion criteria. ${ }^{12}$ Duplicated articles were removed. Two reviewers (KHW and LJC) then independently screened the titles and abstracts. Abstracts providing sufficient details for exclusion were removed. Full texts of potentially eligible studies and those with insufficient details in the abstracts to judge for eligibility were retrieved for further evaluation. We then determined the inclusion of individual studies and resolved any disagreement by discussion among the three reviewers (KHW, LJC, and ALY).

\section{Data extraction and risk of bias assessment}

We extracted the data from studies that met our inclusion criteria. We used a standardized form to record data on the authors, year of publication, country of study, study design, the etiology of DED, sample size, age, gender, duration of disease, the instrument or scale used to assess and grade depression and anxiety, the prevalence and incidence of depression and anxiety, and the score of depression and anxiety based on the scale or instrument used.

Studies were appraised using the STROBE statement. ${ }^{13}$ We assessed the included studies based on the checklist of 22 items that should be addressed in articles reporting on study designs of analytical epidemiology according to the STROBE statement. The qualities of studies were evaluated using the Newcastle-Ottawa Scale. ${ }^{14}$ Two reviewers (KHW and LJC) independently assessed the included studies. In case of discrepancies, a third reviewer (ALY) would arbitrate until a mutual conclusion was reached.

\section{Statistical analysis}

We performed the meta-analysis using the Review Manager software (RevMan, version 5.3; Copenhagen, Denmark: The Nordic Cochrane Centre, The Cochrane Collaboration, 2014). The primary outcomes are the comparisons of the prevalence and the incidence of depression and anxiety between DED and non-DED control subjects. We used the summary Mantel-Haenszel odds ratio (OR) to measure the associations. We also evaluated the severities of depression and anxiety in DED patients, serving as the secondary outcome. As there are different scales and instruments to grade the severity of depression and anxiety, we used the standardized mean difference (SMD) to analyze the results on a uniform scale. SMD is a measure of the size of the intervention effect in each study with respect to the variability within the study and is reported in units of SD. The absolute value is interpreted together with the $P$-value and confidence intervals (CI). ${ }^{15,16}$ We analyzed the statistical heterogeneity of the included studies with the Cochrane Q-statistics $\chi^{2}$-test and $I^{2}$ statistic. If there was significant heterogeneity $(P<0.1)$, a random-effect model was used to pool the data; otherwise a fixed-effect model was adopted.

Studies of DED with any etiologies were pooled and then analyzed as subgroups. The pooling is justified because quality of life and psychological status were comparable between primary Sjogren's syndrome (PSS) and non-SS sicca patients (patients with sicca symptoms with no apparent causes and no features of autoimmunity). ${ }^{17,18} \mathrm{~A}$ recent survey of the members of the Sjögren's Syndrome Foundation revealed that the symptoms of dry eye were the most annoying and activity-limiting aspect of SS. ${ }^{19}$ Furthermore, there was no difference in the degree of depression and anxiety between both groups. ${ }^{20}$ We performed subgroup analysis of the primary and secondary outcomes to identify whether the outcomes would differ between different etiologies of DED. Subgroup analysis was also performed based on the parameters used to define DED, that is, by delineating whether the signs or symptoms alone could account for the plausible association. Finally, the studies are stratified in subgroups by study design to differentiate the prospective studies from the retrospective chart reviews. We conducted a sensitivity analysis to assess whether the primary outcome was robust among different ethnic groups by independently 
assessing the data based on the country of origin in the studies. ${ }^{15}$ We also conducted a sensitivity analysis to test the robustness among different genders, as DED, depression, and anxiety are known to be more prevalent in female patients.

\section{Results}

Figure 1 summarized the process of study inclusion. We identified 711 studies from the literature research. Sixtysix duplicates were removed. We screened the remaining based on their titles and abstracts and excluded another 603 studies. Thus 42 articles were retrieved for full-text review. We excluded 12 studies in which the outcomes were not presented in a quantitative manner, 5 studies in which there was no appropriate control group, 2 studies that did not report relevant outcomes, and 1 study that investigated non-DED patients (ie, fibromyalgia patients). Finally, a total of 22 articles involving 485709 DED patients and 2494317 non-DED subjects were included in the meta-analysis. ${ }^{21-42}$ Of note, no study reported the incidence of depression or anxiety in DED patients, although 'incidence' was among the inclusion criteria.

\section{Characteristics of included studies}

Table 1 described the characteristics of the included studies. They were published between year 1989 and March 2015. The studies were conducted in both Caucasian and Asian populations, including 4 in the United States, 10 in European counties, 7 in the AsianPacific regions, and 1 in Brazil. The study designs included 1 cohort study, 17 case-control studies and 4 cross-sectional studies. The sample sizes in these studies ranged from 36 to 2454458 . Fourteen studies included only PSS patients ${ }^{21-30,35,38,39,41}$ and the remaining eight studies involved DED patients that were defined by an assortment of clinical dry eyes parameters such as Ocular Surface Disease Index, tear break up time, or Schirmer's test. ${ }^{31-34,36,37,40,42}$ Different instruments and scales were used to assess for the presence and the degree of depression and anxiety. Among the 22 included studies, 4 relied on the International Classification of Diseases (ICD-9) coding, while an assorted number of selfadministered psychiatric questionnaires were used to affirm the presence and severity of depression and anxiety in the other studies. On the basis of the STROBE checklist, the quality of the studies in our review was rated from 14 to 19 points on a scale of $0-22$ points (Supplementary Appendix 3). The majority of studies did not adequately address the potential sources of bias, analyze subgroup, or perform sensitivity analysis. The other components from the STROBE checklist were

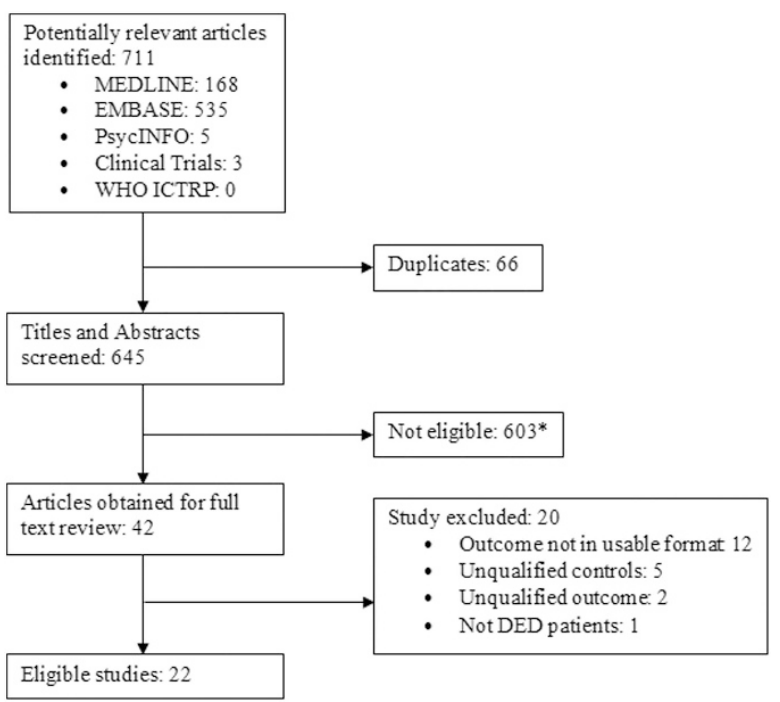

Figure 1 Flow chart showing selection of studies for inclusion in this meta-analysis. * Of these 603 studies, 458 did not involve patients with dry eye disease (DED), depression, or anxiety; 67 studied DED patients, but its association with mental disorder were not its outcome; 27 studied patients with depression or anxiety, but its association with DED were not its outcome; 49 studies were case series or review articles with no control group; 2 studies were not in English.

present across most of the included studies. Overall, the studies scored high on the STROBE checklist are of good quality.

\section{Risk of bias}

Supplementary Appendix 4 summarized the risk of bias assessment using the Newcastle-Ottawa Quality Assessment Scale. All studies had controlled for potential confounders by selecting age- and/or sex-adjusted control groups. The cases were representative with welldefined criteria in the majority of studies. Galor $e a^{33}$ and Van Der Vaart et al ${ }^{42}$ recruited DED patients based on ICD-9 coding and did not perform a baseline assessment of the dry eye status. The ascertainment of depression or anxiety was based on ICD-9 coding or questionnaires conducted by blinded investigators; these questionnaires have been validated to measure the depression and anxiety status in patients. All studies used identical methods to ascertain the outcomes for both the case and control groups. As the studies were retrospective in nature, non-response rate was insignificant.

\section{Outcome measures}

All of the 22 included studies reported an association between DED and depression (13 reported the prevalence, 11 graded the severity, and 2 reported both), 


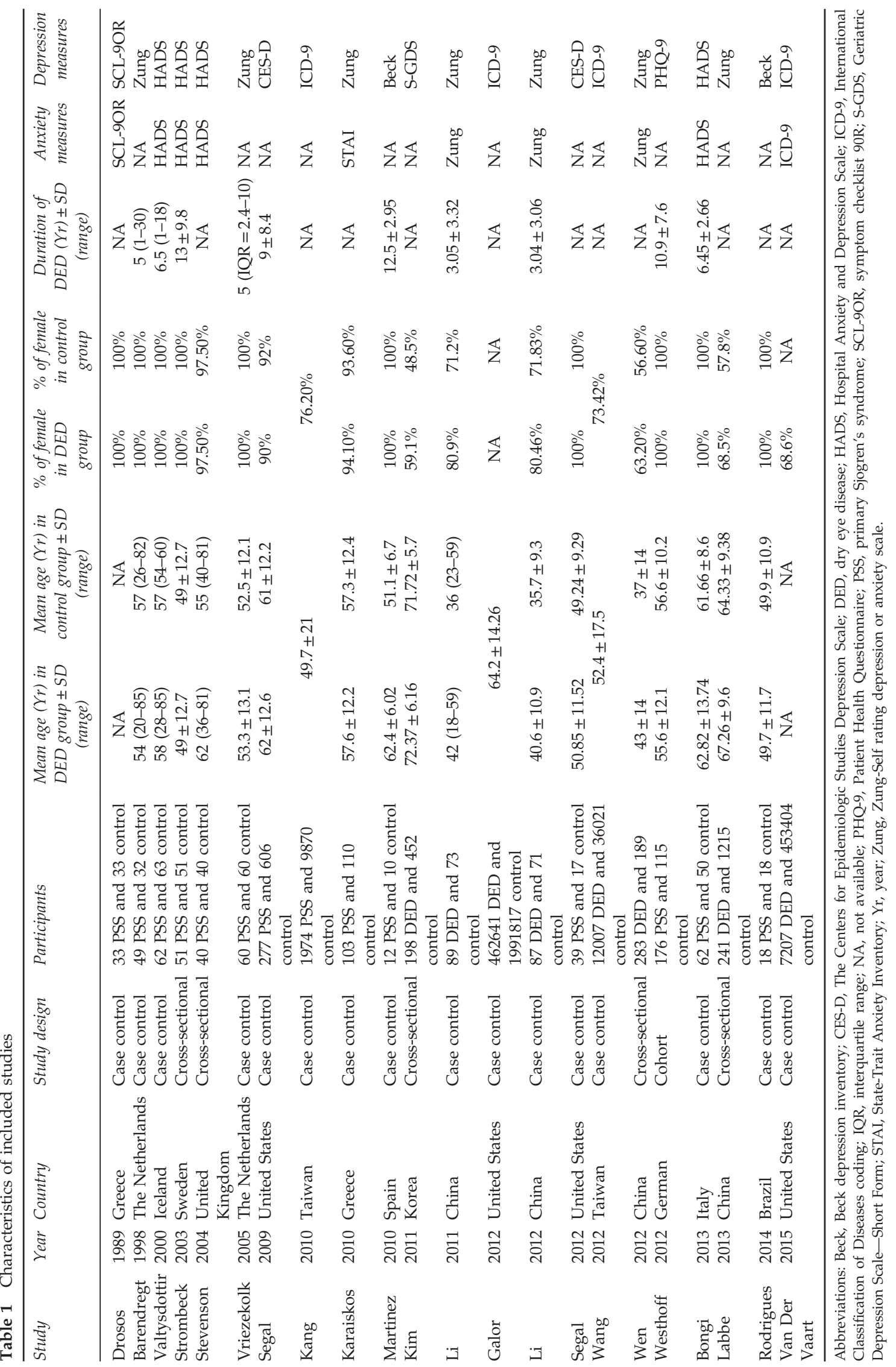


while 10 studies reported an association between DED and anxiety (6 reported the prevalence, 5 graded the severity, and 1 reported both). Meta-analysis showed that
DED was associated with an increased prevalence of depression (summary OR $=2.92,95 \% \mathrm{CI}$ : 2.13-4.01, $P<0.00001, I^{2}=98 \%$ (Figure 2a)) and an increased

a

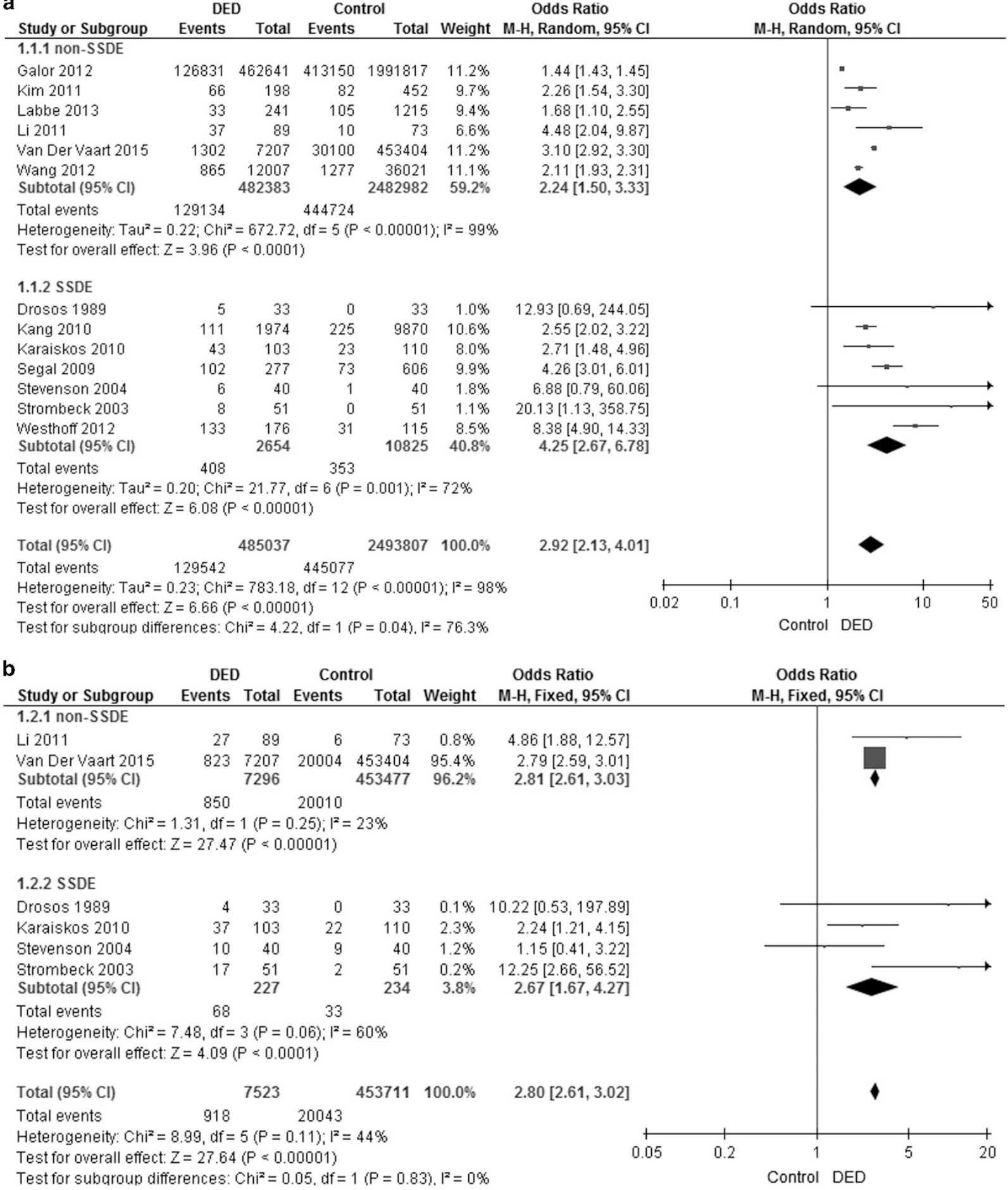

Figure 2 (a) Association between prevalence of depression in dry eye disease (DED) patients versus control group. (b) Association between prevalence of anxiety in DED patients versus control group. CI, confidence interval; M-H, Mantel-Haenszel; SSDE, Sjogren Syndrome Dry Eye. 


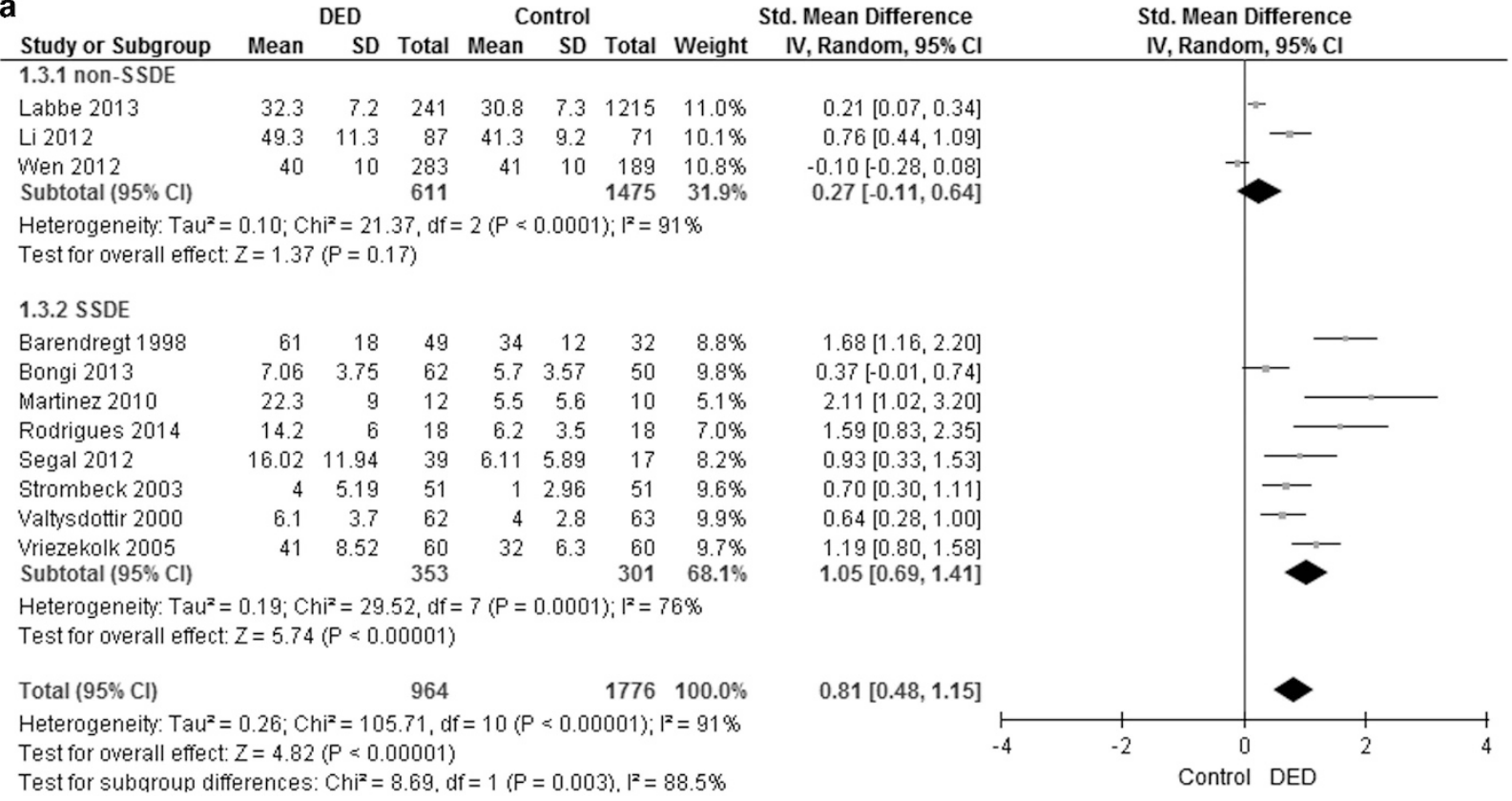

b

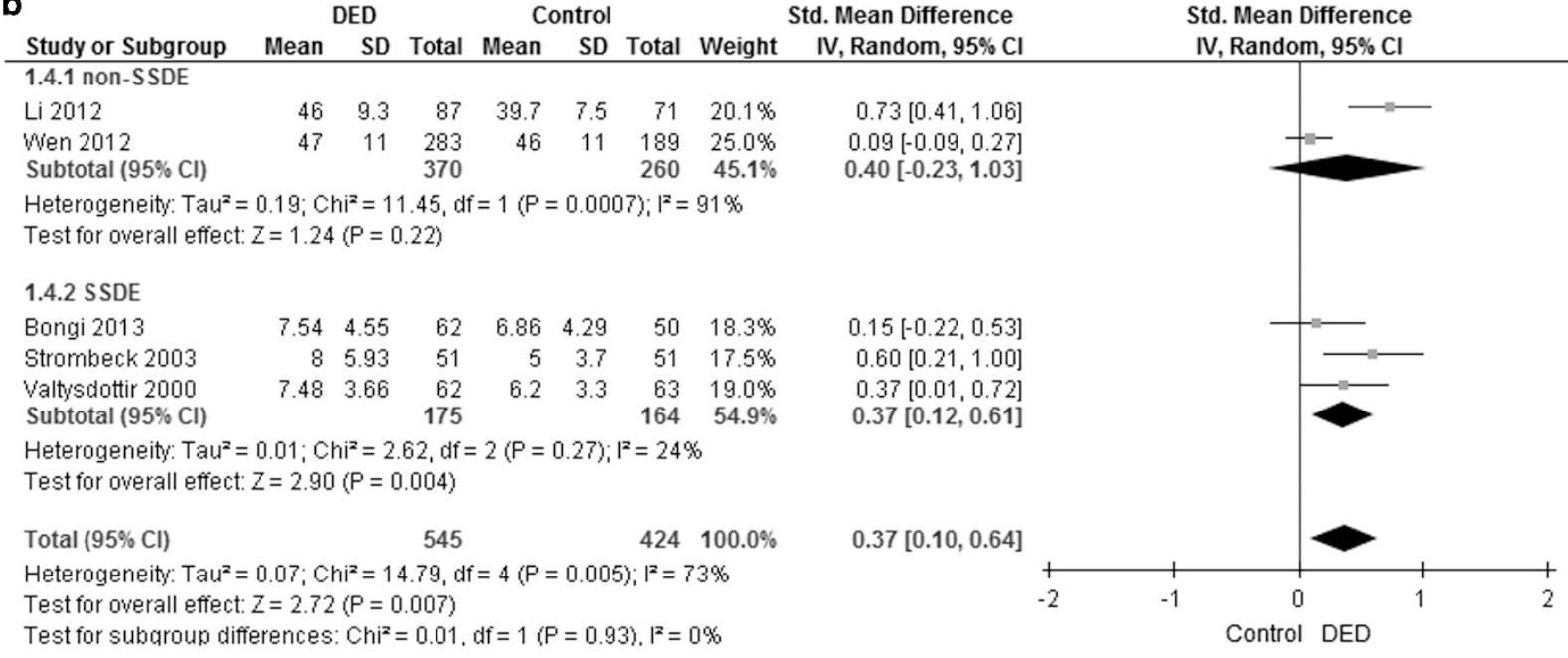

Figure 3 (a) Association between score of depression in dry eye disease (DED) patients versus control group. (b) Association between score of anxiety in DED patients versus control group. CI, confidence interval non-SSDE; IV, inverse variance; SSDE, Sjogren Syndrome Dry Eye; Std. Mean Difference, standardized mean difference.

prevalence of anxiety (summary OR $=2.80,95 \% \mathrm{CI}: 2.61-$ $3.02, P<0.00001, I^{2}=44 \%$ (Figure $2 \mathrm{~b}$ )) as compared with non-DED subjects. The depression scores $(\mathrm{SMD}=0.81$, 95\% CI: $0.48-1.15, P<0.00001, I^{2}=91 \%$ (Figure 3a)) and anxiety scores $(\mathrm{SMD}=0.37,95 \% \mathrm{CI}: 0.10-0.64, P=0.007$, $I^{2}=73 \%$ (Figure $3 b$ )) were greater in DED than non-DED subjects. Statistically significant heterogeneities $\left(I^{2}>50 \%\right)$ were detected in the association between DED and depression, as well as for the assessment of severity in depression and anxiety. All primary and secondary outcomes reached statistical significance. Subgroup analyses demonstrated that the prevalence of depression and anxiety was greater in DED, irrespective of its etiology (Figure 2a and b). The scores of depression and anxiety were greater only in PSS patients (Figure 3a and b). In the included studies, the patients were labeled as having DED by the clinical judgment of the ophthalmologists after assessing the symptoms, clinical signs, and/or auxiliary tests, and not based solely on the signs or symptoms. Thus it was not possible to perform subgroup analysis to differentiate the outcomes based on the signs or symptoms alone. Subgroup analysis by study 
design was not carried out as 21 out of the 22 studies were retrospective. We performed a sensitivity analysis by assessing Caucasian patients or Asian patients alone, and found that our primary outcomes remained unchanged, with significantly greater likelihood of depression and anxiety in DED patients (data not shown). It was not possible to perform a sensitivity analysis to study whether our results would be robust across the different gender as there was insufficient subgroup data.

\section{Discussion}

This is the first systematic review and meta-analysis on the association of DED and mental disorders. Our results confirmed the association of depression and anxiety with DED, irrespective of the underlying etiologies of DED and ethnic differences of the patients. The prevalence of depression and anxiety is approximately threefold higher in DED patients. In particular, DED patients with PSS could suffer from a more severe form of depression and anxiety. Our findings thus suggest that ophthalmologists should be aware of the potential mental comorbidities while managing patients with DED.

\section{Quality of evidence and limitations}

The weight of this association was limited by the clinical, methodological and statistical heterogeneities of the included studies. The DED patients included in our metaanalysis consisted of a spectrum of disease durations and severities. The consistent results in our subgroup analysis based on the underlying etiologies of DED and the sensitivity analysis across difference ethnicities supported the validity the association. As no study reported the incidence, the effects of the duration and severity of DED on the prevalence of depression and anxiety remain unclear. Further longitudinal studies with a long followup period are warranted to address this uncertainty.

The included studies relied on various forms of selfadministered questionnaires and the ICD-9 codes. Although such self-administered questionnaires are common and validated in psychiatric practices, the different scales and cutoffs used to define the presence or the absence of depression and anxiety can vary. Such nature would lead to information bias and methodological heterogeneity when combining these data in a meta-analysis. In view of this issue, we used SMD as an outcome measure to address intra-study variability, which standardizes the results to a uniform scale before they were analyzed. Selection bias was well-controlled by having clear definition of the case and control groups, resulting in comparable baseline characteristics.

Our meta-analysis pooled DED patients of different etiologies. Our subgroup analysis revealed that the prevalence of depression and anxiety was greater in DED patients as compared with healthy controls irrespective of the SS status, while the severity of depression and anxiety was greater in the SS-DED group. This suggests that patients with DED secondary to PSS may have a more severe form of depression and anxiety. However, the numbers of studies that evaluated the severity of depression $(n=3)$ and anxiety $(n=2)$ in non-SS-DED patients were small. Furthermore, in patients labeled as non-SS-DED, they might actually be suffering from SS as a longitudinal cohort of PSS patients had symptoms of dry eyes about 10 years before being diagnosed as PSS. ${ }^{43}$ Thus, these may limit the strength of our interpretation in the subgroup analysis of severity assessment. Although it appears that the rheumatoid symptoms of PSS may affect the psychiatric symptoms, an association study has demonstrated that the prevalence of depression and anxiety in patients with rheumatoid symptoms were the same as healthy controls; ${ }^{23}$ thus we believe that pooling these different etiologies together is justified.

More than half of the included patients and nearly all the patients with PSS were female. This imbalance in gender distribution could have been a confounding factor. Further studies should account for the presence of cohort effects on gender differences by performing subgroup analyses.

\section{Relationship between DED and mental disorders}

Our study revealed that current evidence is largely based on the retrospective chart review. Understanding the cause and effect relationship and the temporal aspect in the reported association warrants further prospective investigations.

Chronic discomfort and pain from dry eye symptoms might negatively affect the cognitive processes, sleep, mood, and mental health. ${ }^{44}$ DED patients also report more frequent chronic pain syndrome with higher pain score with an underlying etiology involving somatization. 8,45 Ocular surface symptoms could negatively impact the performance of daily activities, emotional well-being, and working capacity. ${ }^{46}$ In addition to the irritating ocular symptoms, DED could negatively impact the visual performance and perception of visual function. ${ }^{10}$ The disturbance in visual perception and performance may induce and aggravate the depressive and anxiety symptoms. $3,44,47,48$

Somatization is common in depression, and this could influence the perception of ocular discomfort. ${ }^{49}$ Previous studies reported that depression is more closely correlated with dry eye symptoms but not to dry eye signs, supporting the hypothesis that somatization contributes to the overall dry eye symptoms in 
depressive patients. $6,31,32,40,50$ Although we set out in to perform subgroup analysis to explore whether it is the symptoms or signs of DED that are associated with the mental disorder, the current published literature limited us from doing so. We believe this is important in understanding the pathophysiology behind the association and has important clinical implications on how we think and triage patients with DED. Galor et al ${ }^{6}$ postulated that patients with depression and anxiety may suffer from central sensitization which affects pain perception and pain related behavior. Patients with depression and anxiety interpret ocular sensations differently than healthy controls and the perception of dry eyes symptoms can be influenced by their mood. ${ }^{48}$

Apart from the disease process itself, anti-depressants and anxiolytic are associated with DED due to their potential side effects on the tear film status. ${ }^{51,52}$ The anti-cholinergic effect of these drugs may contribute to dry eye symptoms. ${ }^{53}$ Even in newer generations of anti-depressants, patients using selective serotonin reuptake inhibitors (SSRIs) demonstrated lower Schirmer scores independent of the duration of anti-depressant use as compared with those using serotonin-norepinephrine reuptake inhibitors (SNRIs); and both SSRIs and SNRIs demonstrated an increased risk for DED. ${ }^{54}$

\section{Implications}

The results of our meta-analysis revealed an increased likelihood of depression and anxiety in DED patients across different ethnicities, severities of DED, and types of DED. This is of clinical importance for colleagues involved in the management of DED patients. Ophthalmologists managing DED patients often focus on signs in addressing the patient's leading symptoms and may overlook other comorbidities. Ophthalmologists need to be vigilant and aware of the close link in depression/anxiety and dry eyes in the well-being and functionality of their patients. It would not be practical to screen for depression and anxiety in all DED patients, but those patients with a strong discordance of dry eye signs and symptoms may be the ones more likely to suffer from underlying depression or anxiety. It was demonstrated in our meta-analysis that DED are associated with depression and anxiety but the converse may also be true. Prospective studies that differentiate between the signs and symptoms of DED with a long follow-up duration can help us to understand the causal relationships and the mechanisms underlying the associations of DED with depression and anxiety.

\section{Conclusions}

Our study demonstrated a greater prevalence of depression and anxiety in DED patients and the prevalence and severity of depression are greater in PSS patients. The study highlights the need for prospective research in understanding the directionality and mechanism of the observed associations. Ophthalmologists should be aware of the potential psychiatric comorbidities.

\section{Summary}

What was known before

- Dry eye disease (DED) is one of the most common ophthalmological conditions, and has become a major healthcare problem due to its growing prevalence and impacts on healthcare resources and patients' quality of life.

- There is discordance between the ocular signs and symptoms in dry eye patients.

- The poor quality of life as a result of DED may have a negative impact on the mental health of the patients.

What this study adds

- This meta-analysis consisting of 22 studies with 2,980,026 patients is the first systematic review and meta-analysis to study the association of DED with the prevalence and severity of depression and anxiety.

- The prevalence of depression and anxiety is greater in patients with DED than in controls. In particular, the prevalence and severity of depression is greatest in patients with primary Sjogren's syndrome.

- Ophthalmologists managing dry eye patients should be aware of their potential mental comorbidities in addition to ocular conditions.

\section{Conflict of interest}

The authors declare no conflict of interest.

\section{References}

1 Pflugfelder SC, Geerling G, Kinoshita S, Lemp MA, McCulley J, Nelson D et al. Management and therapy of dry eye disease: report of the Management and Therapy Subcommittee of the International Dry Eye WorkShop (2007). Ocul Surf 2007; 5(2): 163-178.

2 Mertzanis P, Abetz L, Rajagopalan K, Espindle D, Chalmers R, Snyder C et al. The relative burden of dry eye in patients' lives: comparisons to a U.S. normative sample. Invest Ophthalmol Vis Sci 2005; 46(1): 46-50.

3 Miljanovic B, Dana R, Sullivan DA, Schaumberg DA. Impact of dry eye syndrome on vision-related quality of life. Am J Ophthalmol 2007; 143(3): 409-415. 
4 Schein OD, Tielsch JM, Munoz B, Bandeen-Roche K, West S. Relation between signs and symptoms of dry eye in the elderly. A population-based perspective. Ophthalmology 1997; 104(9): 1395-1401.

5 Nichols KK, Mitchell GL, Zadnik K. The repeatability of clinical measurements of dry eye. Cornea 2004; 23(3): 272-285.

6 Galor A, Felix ER, Feuer W, Shalabi N, Martin ER, Margolis TP et al. Dry eye symptoms align more closely to non-ocular conditions than to tear film parameters. Br J Ophthalmol 2015; 99(8): 1126-1129.

7 Vehof J, Sillevis Smitt-Kamminga N, Kozareva D, Nibourg SA, Hammond CJ. Clinical Characteristics of Dry Eye Patients With Chronic Pain Syndromes. Am J Ophthalmol 2016; 162: 59-65 e52.

8 Vehof J, Zavos HM, Lachance G, Hammond CJ, Williams FM. Shared genetic factors underlie chronic pain syndromes. Pain 2014; 155(8): 1562-1568.

9 Vehof J, Wang B, Kozareva D, Hysi PG, Snieder H, Hammond CJ. The heritability of dry eye disease in a female twin cohort. Invest Ophthalmol Vis Sci 2014; 55(11): 7278-7283.

10 Smith JA, Albeitz J, Begley C, Caffery B, Nichols K, Schaumberg $\mathrm{D}$ et al. The epidemiology of dry eye disease: report of the Epidemiology Subcommittee of the International Dry Eye WorkShop (2007). Ocul Surf 2007; 5(2) 93-107.

11 Stroup DF, Berlin JA, Morton SC, Olkin I, Williamson GD, Rennie D et al. Meta-analysis of observational studies in epidemiology: a proposal for reporting. Meta-analysis Of Observational Studies in Epidemiology (MOOSE) group. JAMA 2000; 283(15): 2008-2012.

12 Chapter 7: Selecting studies and collecting data In: Higgins JPT, Deeks JJ, Altman DG, eds. Cochrane Handbook of Systematic Reviews of Interventions. Version 5.1.0 [updated March 2011]: The Cochrane Collaboration, 2011. Available from: www.cochrane-handbook.org. Accessed on 20 November 2015.

13 von Elm E, Altman DG, Egger M, Pocock SJ, Gotzsche PC, Vandenbroucke JP. The Strengthening the Reporting of Observational Studies in Epidemiology (STROBE) statement: guidelines for reporting observational studies. Lancet 2007; 370(9596): 1453-1457.

14 Wells GA, Shea B, O'Connell D, Peterson J, Welch V, Losos $\mathrm{M}$ et al. The Newcastle-Ottawa scale (NOS) for assessing the quality of nonrandomized studies in metaanalysis. The Ottawa Health Research Institute. Available from: http://www.ohri.ca/programs/clinical_epidemiology/ oxford.asp. Accessed on 14 March 2015.

15 Chapter 9: Analysing data and undertaking meta-analyses. In: Deeks JJ, Higgins JPT, Altman DG (eds). Cochrane Handbook of Systematic Reviews of Interventions, Version 5.1.0 [updated March 2011]. The Cochrane Collaboration, 2011. Available from: http:/ / www.cochrane-handbook.org (accessed on 14 June 2015).

16 Wan KH, Chen LJ, Rong SS, Pang CP, Young AL. Topical cyclosporine in the treatment of allergic conjunctivitis: a meta-analysis. Ophthalmology 2013; 120(11): 2197-2203.

17 Rostron J, Rogers S, Longman L, Kaney S, Field EA. Healthrelated quality of life in patients with primary Sjogren's syndrome and xerostomia: a comparative study. Gerodontology 2002; 19(1): 53-59.

18 Champey J, Corruble E, Gottenberg JE, Buhl C, Meyer T, Caudmont C et al. Quality of life and psychological status in patients with primary Sjogren's syndrome and sicca symptoms without autoimmune features. Arthritis Rheum 2006; 55(3): 451-457.

19 Foulks GN, Forstot SL, Donshik PC, Forstot JZ, Goldstein MH, Lemp MA et al. Clinical guidelines for management of dry eye associated with Sjogren disease. Ocul Surf 2015; 13(2): 118-132.

20 Cho HJ, Yoo JJ, Yun CY, Kang EH, Lee HJ, Hyon JY et al. The EULAR Sjogren's syndrome patient reported index as an independent determinant of health-related quality of life in primary Sjogren's syndrome patients: in comparison with non-Sjogren's sicca patients. Rheumatology (Oxford) 2013; 52(12): 2208-2217.

21 Drosos AA, Angelopoulos NV, Liakos A, Moutsopoulos HM. Personality structure disturbances and psychiatric manifestations in primary Sjogren's syndrome. J Autoimmun 1989; 2(4): 489-493.

22 Barendregt PJ, Visser MR, Smets EM, Tulen JH, van den Meiracker AH, Boomsma F et al. Fatigue in primary Sjogren's syndrome. Ann Rheum Dis 1998; 57(5): 291-295.

23 Valtysdottir ST, Gudbjornsson B, Lindqvist U, Hallgren R, Hetta J. Anxiety and depression in patients with primary Sjogren's syndrome. J Rheumatol 2000; 27(1): 165-169.

24 Strombeck B, Ekdahl C, Manthorpe R. Jacobsson LTH. Physical capacity in women with primary Sjogren's syndrome: a controlled study. Arthritis Rheum 2003; 49(5): 681-688.

25 Stevenson HA, Jones ME, Rostron JL, Longman LP, Field EA. UK patients with primary Sjogren's syndrome are at increased risk from clinical depression. Gerodontology 2004; 21(3): 141-145.

26 Vriezekolk JE, Geenen R, Hartkamp A, Godaert GLR, Bootsma H, Kruize AA et al. Psychological and somatic predictors of perceived and measured ocular dryness of patients with primary Sjogren's syndrome. J Rheumatol 2005; 32(12): 2351-2355.

27 Segal B, Bowman SJ, Fox PC, Vivino FB, Murukutla N, Brodscholl J et al. Primary Sjogren's Syndrome: health experiences and predictors of health quality among patients in the United States. Health Qual Life Outcomes 2009; 7: 46.

28 Kang J-H, Lin H-C. Comorbidities in patients with primary Sjogren's syndrome: a registry-based case-control study. J Rheumatol 2010; 37(6): 1188-1194.

29 Karaiskos D, Mavragani CP, Sinno MH, Dechelotte P, Zintzaras E, Skopouli FN et al. Psychopathological and personality features in primary Sjogren's syndromeassociations with autoantibodies to neuropeptides. Rheumatology 2010; 49(9): 1762-1769.

30 Martinez S, Caceres C, Mataro M, Escudero D, Latorre P, Davalos A. Is there progressive cognitive dysfunction in Sjogren Syndrome? A preliminary study. Acta Neurol Scand 2010; 122(3): 182-188.

31 Kim KW, Han SB, Han ER, Woo SJ, Lee JJ, Yoon JC et al. Association between depression and dry eye disease in an elderly population. Invest Ophthalmol Vis Sci 2011; 52(11): 7954-7958.

32 Li M, Gong L, Sun X, Chapin WJ. Anxiety and depression in patients with dry eye syndrome. Curr Eye Res 2011; 36(1): $1-7$.

33 Galor A, Feuer W, Lee DJ, Florez H, Faler AL, Zann KL et al. Depression, post-traumatic stress disorder, and dry eye syndrome: a study utilizing the national United States Veterans Affairs administrative database. Am J Ophthalmol 2012; 154(2): 340-346.e342. 
34 Li M, Gong L, Chapin WJ, Zhu M. Assessment of visionrelated quality of life in dry eye patients. Invest Ophthalmol Vis Sci 2012; 53: 5722-5727.

35 Segal BM, Pogatchnik B, Holker E, Liu H, Sloan J, Rhodus N et al. Primary Sjogren's syndrome: cognitive symptoms, mood, and cognitive performance. Acta Neurol Scand 2012; 125(4): 272-278.

36 Wang TJ, Wang IJ, Hu CC, Lin HC. Comorbidities of dry eye disease: a nationwide population-based study. Acta Ophthalmol 2012; 90(7): 663-668.

37 Wen W, Wu Y, Chen Y, Gong L, Li M, Chen X et al. Dry eye disease in patients with depressive and anxiety disorders in Shanghai. Cornea 2012; 31(6): 686-692.

38 Westhoff G, Dorner T, Zink A. Fatigue and depression predict physician visits and work disability in women with primary Sjogren's syndrome: results from a cohort study. Rheumatology 2012; 51(2): 262-269.

39 Bongi SM, Rosso AD, Orlandi M, Matucci-Cerinic M. Gynaecological symptoms and sexual disability in women with primary sjogren's syndrome and sicca syndrome. Clin Exp Rheumatol 2013; 31(5): 683-690.

40 Labbe A, Wang YX, Jie Y, Baudouin C, Jonas JB, Xu L. Dry eye disease, dry eye symptoms and depression: the Beijing Eye Study. Br J Ophthalmol 2013; 97(11): 1399-1403.

41 Rodrigues D-N, Hora JSI, Salgado MCF, Paes RA, Vasconcelos CCF, Landeira-Fernandez $\mathrm{J}$ et al. A short neuropsychological evaluation of patients with primary Sjogren's syndrome. Arq Neuropsiquiatr 2014; 72(1): 38-43.

42 Van Der Vaart R, Weaver MA, Lefebvre C, Davis RM. The association between dry eye disease and depression and anxiety in a large population-based study. Am J Ophthalmol 2015; 159(3): 470-474.

43 Akpek EK, Mathews P, Hahn S, Hessen M, Kim J, GraderBeck $\mathrm{T}$ et al. Ocular and systemic morbidity in a longitudinal cohort of Sjogren's syndrome. Ophthalmology 2015; 122(1): 56-61.
44 Fine PG. Long-term consequences of chronic pain: mounting evidence for pain as a neurological disease and parallels with other chronic disease states. Pain Med 2011; 12 (7): 996-1004.

45 Galor A, Covington D, Levitt AE, McManus KT, Seiden B, Felix ER et al. Neuropathic ocular pain due to dry eye is associated with multiple comorbid chronic pain syndromes. J Pain 2015; 17(3): 310-318.

46 Pouyeh B, Viteri E, Feuer W, Lee DJ, Florez H, Fabian JA et al. Impact of ocular surface symptoms on quality of life in a United States veterans affairs population. Am J Ophthalmol 2012; 153(6): 1061-1066.e1063.

47 Denoyer A, Rabut G, Baudouin C. Tear film aberration dynamics and vision-related quality of life in patients with dry eye disease. Ophthalmology 2012; 119(9): 1811-1818.

48 Kawashima M, Uchino M, Yokoi N, Uchino Y, Dogru M, Komuro A et al. Associations between subjective happiness and dry eye disease: A New Perspective from the Osaka Study. PLoS One 2015; 10(4): e0123299.

49 Katon W, Kleinman A, Rosen G. Depression and somatization: a review. Part I. Am J Med 1982; 72(1): 127-135.

50 Fernandez CA, Galor A, Arheart KL, Musselman DL, Venincasa VD, Florez HJ et al. Dry eye syndrome, posttraumatic stress disorder, and depression in an older male veteran population. Invest Ophthalmol Vis Sci 2013; 54(5): 3666-3672.

51 Moss SE, Klein R, Klein BE. Long-term incidence of dry eye in an older population. Optom Vis Sci 2008; 85(8): 668-674.

52 Lemp MA. Advances in understanding and managing dry eye disease. Am J Ophthalmol 2008; 146(3): 350-356.e351.

53 Wong J, Lan W, Ong LM, Tong L. Non-hormonal systemic medications and dry eye. Ocul Surf 2011; 9(4): 212-226.

54 Kocer E, Kocer A, Ozsutcu M, Dursun AE, Krpnar I. Dry eye related to commonly used new antidepressants. J Clin Psychopharmacol 2015; 35(4): 411-413.

Supplementary Information accompanies this paper on Eye website (http://www.nature.com/eye) 